\title{
Effects of long-term ethanol consumption in mice: interaction between BDNF and brain serotonin systems
}

\author{
Naumenko V.S. \\ Institute of Cytology and Genetics \\ SB RAS, Novosibirsk, Russia \\ naumenko2002@mail.ru \\ Bazovkina D.V \\ Institute of Cytology and Genetics \\ SB RAS, Novosibirsk, Russia \\ daryabazovkina@gmail.com
}

\author{
Ilchibaeva T.V. \\ Institute of Cytology and Genetics \\ SB RAS, Novosibirsk, Russia \\ rbicehok@mail.ru \\ Popova N.K. \\ Institute of Cytology and Genetics \\ SB RAS, Novosibirsk, Russia \\ npopova@bionet.nsc.ru
}

\author{
Antonov Y.V. \\ Institute of Cytology and Genetics \\ SB RAS, Novosibirsk, Russia \\ yegor@bionet.nsc.ru
}

\begin{abstract}
Brain serotonin (5-HT) and brain-derived neurotrophic factor (BDNF) are principal players in the mechanisms of brain and behavioral plasticity. Here we investigated the effect of chronic (6 weeks) consumption of $10 \%$ alcohol on the principal elements of BDNF (BDNF, proBDNF, p75 and TrkB receptors) and 5-HT (5-HT, 5HIAA, tryptophan hydroxylase-2 (Tph-2), 5-HT transporter (5-HTT), 5-HT1A, 5-HT2A and 5-HT7 receptors) systems in the brain of $\mathrm{C} 57 \mathrm{BI} / 6$ mice. BDNF mRNA level in the midbrain and BDNF protein level in the hippocampus were lowered in ethanol-treated mice. The increase in proBDNF protein level in the midbrain, cortex, and amygdala and the increase of $\mathrm{p} 75$ receptor protein level in the midbrain were revealed after ethanol exposure. Alcohol intake reduced the protein level and increased the activity of Tph-2, the key enzyme for serotonin biosynthesis in the brain, and increased the main 5HT metabolite 5-HIAA level and 5-HIAA/5-HT ratio as well as 5-HT7 receptor mRNA level in the midbrain. In the cortex, 5-HT2A receptor protein level was reduced, and 5-HIAA/5HT ratio was increased. Alcohol-induced changes in BDNF and 5-HT systems were revealed in the midbrain where the majority of the cell bodies of the 5-HT neurons are localized, as well as in the cortex, hippocampus and amygdala. Our data allow suggesting that BDNF/5-HT interaction contribute to the mechanism underlying chronic alcohol-induced neurodegenerative disorders.
\end{abstract}

Keywords - alcohol consumption, brain serotonin system, brain-derived neurotrophic factor, mice

Brain serotonin (5-HT) and brain-derived neurotrophic factor (BDNF) are principal players in the mechanisms of brain and behavioral plasticity. The brain 5-HT system is involved in the regulation of wide range of physiological functions and different kinds of normal and disordered behavior. The incredible polyfunctionality of 5-HT is mediated by 14 different 5-HT receptor subtypes expressed in the mammals. In its turn, BDNF is crucially involved in the mechanisms of growth, development, functioning of the nervous system and in the response to stressful events. BDNF is notable by structural and functional complexity. Mature BDNF is produced by proteolytic cleavage of precursor, proBDNF. BDNF precursor proBDNF is biologically active inducing oppose to the mature BDNF effects via p75 receptor. Whereas mature BDNF plays crucial role in the neurogenesis promoting cell survival and differentiation via TrkB receptors, BDNF precursor via $\mathrm{p} 75$ initiates receptors apoptosis. Accumulating evidence indicates the cross-talk between 5-HT and BDNF systems and the crucial role of this interaction in the regulation of brain functioning.
The aim of the present study was to investigate the effect of chronic alcohol consumption on the principal elements of BDNF (BDNF, proBDNF, p75 and TrkB receptors) and 5HT (5-HT, 5-HIAA, tryptophan hydroxylase-2 (Tph-2), 5HT transporter (5-HTT), 5-HT1A, 5-HT2A and 5-HT7 receptors) systems in the brain of $\mathrm{C} 57 \mathrm{Bl} / 6$ mice.

Experiments were carried out on C57BL/6j mice. Mice were treated with $10 \%(\mathrm{w} / \mathrm{v})$ ethanol solution in drinking water (one bottle without choice) for a period of six weeks. mRNA levels were assessed using quantitative real-time RT-PCR technique. Protein levels were estimated by Western-Blot analysis. High Performance Liquid Chromatography was applied for 5-HT and its metabolite 5hydroxiindol acetic acid (5-HIAA) quantification as well as for Tph2 activity estimation. The data were analyzed using one-way ANOVA followed by Fisher's LSD post-hoc comparison.

The chronic alcohol consumption induced reduction of BDNF mRNA level in the midbrain and BDNF protein level in the hippocampus. However, it failed to affect the expression of gene encoding TrkB receptor as well as TrkBFL and TrkB-T1 receptor protein levels. At the same time, the increase in proBDNF protein level in the midbrain, frontal cortex, and amygdala and the increase of p75 receptor protein level in the midbrain raphe nuclei area were revealed after ethanol exposure. Chronic alcohol intake reduced the protein level and increased the activity of Tph2, the key enzyme for serotonin biosynthesis in the brain. These changes were accompanied by increased 5-HIAA level and 5-HIAA/5-HT ratio as well as 5-HT7 receptor mRNA level in the midbrain. In the frontal cortex, 5-HT2A receptor protein level was reduced, and 5-HIAA/5-HT ratio was increased.

These data showed adverse impact of alcoholization on the BDNF system linked with pro-apoptotic effect of alcohol-induced proBDNF and p75 receptor activation. Pronounced alcohol-induced changes in both BDNF and 5HT systems were revealed in the raphe nuclei area of the midbrain where the majority of the cell bodies of the 5-HT neurons are localized. Our data allow suggesting that $\mathrm{BDNF} / 5-\mathrm{HT}$ interaction contribute to the mechanism underlying chronic alcohol-induced effects.

The cost of animal maintenance was supported by the basic research project \# 0324-2019-0041-C-01; the study was supported by the Russian Scientific Foundation (grant No 19-15-00025). 